\title{
AVALIAÇÃO HUMANA DA TRADUÇÃO AUTOMÁTICA DE COMBINAÇÕES LEXICAIS ESPECIALIZADAS: O CASO DO GOOGLE TRANSLATE E DO DEEPL
}

\author{
HUMAN EVALUATION OF MACHINE TRANSLATION OF SPECIALIZED \\ LEXICAL COMBINATIONS: TESTING GOOGLE TRANSLATE AND DEEPL
}

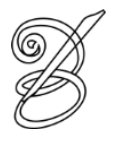

\author{
Thais Miranda e BORGES \\ Pesquisadora autônoma \\ Rio de Janeiro, Rio de Janeiro, Brasil \\ orcid.org/0000-0001-7092-7424 \\ mirandaeborges.thais@gmail.com
}

\author{
Janine Maria Mendonça PIMENTEL \\ Universidade Federal do Rio de Janeiro \\ Faculdade de Letras \\ Departamento de Anglo-Germânicas \\ Rio de Janeiro, Rio de Janeiro, Brasil \\ orcid.org/0000-0001-6576-9898 \\ janine.m.pimentel@gmail.com
}

Resumo: Este trabalho se propõe a avaliar a tradução automática feita pelos sistemas Google Translate e DeepL especificamente em relação a combinações lexicais especializadas (L'HOMME; BERTRAND, 2000) compostas por verbo+substantivo extraídas de textos jurídicos chamados "cláusulas arbitrais". Nós apresentamos uma metodologia para selecionar as combinações lexicais especializadas e avaliar a adequação e a fluência das traduções produzidas pelos sistemas em questão. Os resultados obtidos estão alinhados com recentes pesquisas na área de tradução automática baseada em redes neurais (NMT) que indicam que esse tipo de tecnologia tem um desempenho melhor no parâmetro "fluência" do que no parâmetro "adequação" (KOEHN; KNOWLES, 2017). Em relação à precisão terminológica, houve ocorrências de tradução de termos diferentes na língua-fonte para o mesmo termo na língua-alvo em uma mesma frase, o que muitas vezes pode ser inadequado em textos técnicos e contraria a percepção bastante comum de que a tradução automática produz resultados consistentes, sendo mais adequada à tradução especializada. Ainda assim, e apesar das limitações apontadas neste estudo, as avaliações das traduções das combinações lexicais foram bastante satisfatórias.

Palavras-chave: Tradução automática. Textos jurídicos. Combinações lexicais especializadas. Avaliação da tradução.

\begin{abstract}
This study evaluates the translations of specialized lexical combinations (L'HOMME; BERTRAND, 2000) produced by two different machine translation systems, i.e., Google Translate and DeepL. The selected lexical combinations have a verb+noun structure and were extracted from a legal genre known as "arbitration clauses". A methodology for selecting the specialized lexical combinations and evaluating the adequacy and fluency of the translations is presented. The results are in line with recent research in the field of neural machine translation (NMT) suggesting that the outputs of this type of machine translation system are more "fluent" than "adequate" (KOEHN; KNOWLES, 2017). As for terminological accuracy, different terms in the source language received the same translation in the target language within the same sentence, which contradicts the idea that machine translation generates consistent results and is, thus, more appropriate for specialized translation. Nevertheless, despite the shortcomings discussed in the study, the translations of the lexical combinations were considered quite satisfactory.
\end{abstract}

Keywords: Machine translation. Legal texts. Specialized lexical combinations. Translation evaluation. 


\section{Introdução}

$\mathrm{E}$

ste trabalho se propõe a realizar uma avaliação de qualidade da tradução automática produzida por sistemas atuais para verificar em que medida estes podem ser úteis como mais uma ferramenta à disposição dos tradutores. Sendo essa uma tarefa muitíssimo abrangente, pretendemos oferecer uma pequena contribuição, com a esperança de fornecer subsídios para informar a decisão dos tradutores a respeito do uso de sistemas de tradução automática. Nossa contribuição consiste na avaliação da tradução automática feita pelos sistemas Google Translate e DeepL especificamente em relação a combinações lexicais especializadas compostas por verbo+substantivo extraídas de textos jurídicos chamados "cláusulas arbitrais".

Muitos avanços foram feitos nos últimos anos no que diz respeito à qualidade da tradução automática, em grande parte devido ao lançamento de tecnologia de redes neurais como base de tais sistemas, conhecida pela sigla NMT (em inglês, Neural Machine Translation). Sendo a NMT considerada atualmente o estado da arte, a Microsoft afirma que ela atinge "a igualdade com os humanos" (HASSAN et al., 2018, p. 2, tradução nossa) ${ }^{1}$ no 22 trabalho da tradução, e a Google defende que essa tecnologia é capaz de "superar a lacuna entre a tradução humana e a tradução automática" (WU et al., 2016, p. 1, tradução nossa)². Contudo, é necessário analisar cuidadosamente se há, de fato, avanços e ganhos ao tradutor individual no uso de tais sistemas.

A motivação da pesquisa que apresentamos aqui surgiu da observação do contexto atual mundial em que muito se fala na 4⿳⺈冂 Revolução Industrial, marcada pela automação, pela digitalização bem como pelas transformações que estão ocorrendo - e vão ocorrer - em todos os mercados de trabalho. Estando o tradutor inserido também nesse contexto, é preciso que ele esteja a par dos avanços na sua área de trabalho para não se tornar um agente meramente passivo, mas ter voz nessa transformação: seja para defender seu valor como profissional, para ser capaz de se adaptar mais rapidamente às mudanças no mercado, para estar apto a usar a tecnologia em seu benefício aumentando sua produtividade ou mesmo para ampliar seu campo de trabalho, por exemplo, em colaborações necessárias - mas ainda subestimadas entre tradutores e desenvolvedores de tecnologia.

O presente artigo está organizado da seguinte forma. Depois de definirmos o nosso objeto de avaliação - as combinações lexicais especializadas (seção 2) -, traçamos um breve estado da arte sobre as pesquisas na área da avaliação da qualidade da tradução automática (seção 3). Em seguida, apresentamos uma metodologia para selecionar as combinações 
lexicais especializadas e comparar as traduções que o Google Translate e o DeepL oferecem (seção 4). Por fim, apresentamos os resultados obtidos (seção 5) e as conclusões que podemos tirar a partir da análise das traduções (seção 6).

\section{Combinações lexicais especializadas}

$\mathrm{Na}$ interação cotidiana, combinações linguísticas convencionais são usadas frequentemente pelos falantes nativos para exprimir determinada ideia ou conceito (RAMISCH, 2009). O sentido expresso por essa combinação se destaca por não ser meramente a soma do significado de cada uma das palavras que compõe a combinação, mas por transmitir uma mensagem consolidada. Não é possível compreender o significado da expressão "engolir um sapo" de forma literal, mas seus termos combinados transmitem o sentido de suportar desaforos sem revidar. Tampouco é possível substituir por sinônimos o segundo termo de "lua cheia", pois "lua completa" ou "lua preenchida" não seriam adequados em português. Tais combinações, genericamente denominadas expressões multipalavras, incluem fenômenos diversos e, ao mesmo tempo, estreitamente relacionados - por exemplo, as colocações, os verbos frasais e as expressões idiomáticas (VILLAVICENCIO; RAMISCH, 2010).

No domínio especializado, há combinações de palavras que ocorrem com frequência elevada e, portanto, trazem naturalidade ao discurso (WAQUIL, 2013). É essencial que o tradutor especializado conheça a convencionalidade da área em que atua para que possa produzir traduções com linguagem similar à dos especialistas, que são em geral seu públicoalvo. Segundo L'Homme e Bertrand (2000), muitos estudiosos das combinações de palavras em domínios especializados frequentemente se referem a elas como colocações, mas ainda não foi demonstrado que as combinações lexicais especializadas se comportam da mesma forma que as colocações da língua geral. Com o intuito de diferenciá-las, as autoras adotam a denominação "colocações" para se referirem às combinações próprias da língua geral e “combinações lexicais especializadas" para aquelas pertencentes a um domínio especializado.

O estudo de L'Homme e Bertrand (2000) destaca a importância dos coocorrentes, que são frequentemente negligenciados (WAQUIL, 2013). Com base em análises feitas por outros autores, as pesquisadoras ressaltam que os coocorrentes podem se combinar com outras unidades terminológicas (L'HOMME; BERTRAND, 2000) e adotam a divisão que Heid (2014) faz das combinações lexicais especializadas: colocações lexicais (o coocorrente se combina com apenas uma unidade terminológica) e colocações conceituais (o coocorrente se 
combina com várias unidades terminológicas). Esse estudo evoluiu para um modelo de descrição de verbos especializados cuja aplicação em corpora verificou que determinados verbos se combinam frequentemente com várias unidades terminológicas que compartilham propriedades semânticas (L'HOMME; BERTRAND, 2000). A metodologia proposta pelas autoras envolve as seguintes etapas: (1) seleção de palavras-chave preliminares (e.g., aéronef), (2) extração de combinações lexicais em que as palavras-chave preliminares são usadas (e.g., aéronef décolle, exploiter un aéronef, exploitation d'un aéronaf), (3) seleção dos coocorrentes e nova extração de combinações com os coocorrentes selecionados (e.g., avion décolle, giravion décolle), (4) estudo das combinações extraídas.

O trabalho que aqui apresentamos utiliza a metodologia proposta por L'Homme e Bertrand (2000) para selecionar as combinações lexicais especializadas compostas por verbo+substantivo extraídas de um pequeno corpus de cláusulas arbitrais. Tendo em vista que o objetivo deste trabalho foi a avaliação da qualidade do texto resultante de tradução automática, a etapa (4) desse modelo não foi utilizada.

\section{Avaliação da qualidade da tradução automática}

Embora a noção de erro não tenha limites precisos na área dos Estudos da Tradução, podendo ser analisada por diferentes perspectivas (FROTA, 2006), há certo consenso sobre alguns parâmetros do fazer tradutório, dentre eles a fluência do texto-alvo. Conforme observa Munday (2012), por exemplo, a certificação de tradutores mais conhecida do Reino Unido, Chartered Institute of Linguists (CIOL), valoriza traduções que soem natural na língua-alvo. Por estarem relacionadas à convencionalidade da língua e, portanto, ao que é usual para uma comunidade de falantes, as combinações lexicais especializadas têm importância fundamental na tradução, uma vez que são determinantes para a fluência e a naturalidade do texto-alvo.

A combinação convencional de palavras é um fenômeno que apresenta desafios não só para a área da terminologia, lexicografia e tradução (ORENHA, 2009; TAGNIN, 2002), mas também para a área de processamento de linguagem natural em sua aplicação à tradução automática. Enquanto Pérez-Hernández et al. (1999) consideram os "hábitos colocacionais" como um dos cavalos de batalha da lexicografia, Sag et al. (2002) se referem às expressões multipalavras como "pain in the neck"3 para o processamento de linguagem natural.

São muitas as dificuldades que as combinações lexicais apresentam para a tradução automática e, embora sua importância para essa área tenha sido reconhecida, apenas recentemente foi dada maior atenção aos desafios que elas impõem. Primeiramente, as 
combinações lexicais são muitíssimo frequentes e há poucos recursos lexicais bilíngues ou multilíngues que as contemplem (L'HOMME; BERTRAND, 2000). Em segundo lugar, as palavras que as compõem nem sempre estão uma ao lado da outra, mas podem aparecer separadas (ficar mesmo a ver navios; tomar um longo banho; bater o maior papo). Em terceiro lugar, seu grau de composicionalidade é variável, podendo haver combinações lexicais livres (água limpa) e fixas (peixe fora d'água). Em quarto lugar, suas características morfossintáticas podem permitir variações na sua forma (no contexto jurídico, por exemplo: parte interveniente, partes intervenientes). Em quinto lugar, por não serem composicionais, sua tradução nem sempre será literal (se traduzida literalmente para o português, a expressão kick the bucket resultará em "chutar o balde", que - embora exista - não significa morrer como em inglês, mas desistir; uma tradução possível - e não literal - com o mesmo significado seria "bater as botas"). Por último, tem-se que nem todas as combinações lexicais possuem as mesmas características semânticas e sintáticas - o que dificulta ainda mais sua identificação e tradução (CONSTANT et al., 2017).

Na tentativa de explicar por que a tradução é uma tarefa difícil para os computadores, Arnold (2003) afirma que parte dessa dificuldade vem do fato de ser a tradução, por si só, uma tarefa difícil até mesmo para seres humanos, pois envolve habilidades multifacetadas que exigem muito mais do que simplesmente saber duas línguas. Ao detalhar os problemas enfrentados pelos computadores ao traduzir, o autor inclui a incapacidade da máquina de aplicar o senso comum, que envolve literalmente milhões de fatos sobre o mundo. Para a tradução das combinações lexicais, é esse conhecimento de mundo - mais precisamente o conhecimento cultural quanto ao uso da língua - que será essencial.

Inicialmente, a avaliação dos resultados da tradução automática era uma atividade que necessariamente envolvia um trabalho humano e, como tal, implicava custos, tempo e subjetividade. Com o intuito de eliminar essas questões, foram desenvolvidas métricas de avaliação de tradução automática, dentre elas a métrica BLEU (FIEDERER; O'BRIEN, 2009). Segundo Fiederer e O’Brien, a BLEU tem como premissa a ideia de que, quanto mais próximo o resultado da tradução automática estiver de uma tradução humana profissional, melhor ele será. Essa 'proximidade' é avaliada por medidas estatísticas que, curiosamente, não são independentes dos humanos, pois precisam de um padrão de tradução humana chamado gold standard - com o qual os resultados da tradução automática são comparados (FIEDERER; O’BRIEN, 2009). De acordo com as autoras, há, contudo, muita discussão a respeito da verdadeira correlação entre o alto resultado de tais métricas e o padrão humano. A 
falta de uma metodologia única para a avaliação de traduções gera bastante controvérsia (MARTÍN, 2017), sendo fonte de intensas discordâncias entre a academia e a indústria (CASTILHO; O’BRIEN, 2017). Existem diversas metodologias que se utilizam de diferentes parâmetros, mas todas têm o mesmo objetivo genérico: avaliação da qualidade. Dessa forma, para a escolha da metodologia de avaliação, é importante assegurar sua adequação ao objetivo específico da tarefa - por exemplo, comparação entre a tradução de colocações por sistemas de tradução automática - e, ainda, definir o que será considerado como qualidade.

Em uma definição básica de qualidade, Muzii (2006) ressalta a importância de se atentar para a necessidade do usuário ao definir qualidade, de forma genérica, como "uma integração de características que determinam em que medida os resultados suprem as necessidades do cliente" (MUZII, 2006, p. 19, tradução nossa) ${ }^{4}$. Além da figura do cliente, que pode ter exigências específicas de qualidade, há que se considerar o propósito da tradução, ou seja, para que finalidade a tradução será usada. Por esse motivo, há várias formas de conduzir a avaliação humana da qualidade das traduções. Uma delas é a classificação (ranking). Nesse caso, o avaliador humano classifica traduções feitas por mais de um sistema (ou por sistemas e tradutores humanos) em ordem de qualidade. Outra forma de avaliação é em relação à adequação (adequacy). Para tal, o avaliador é orientado a verificar até que ponto a tradução transfere o significado do texto-fonte para o texto-alvo e a expressar a análise em uma escala Likert (CASTILHO et al., 2018). A análise é, portanto, centrada na mensagem, ou seja, refere-se à semântica. Há, também, a avaliação que prioriza a fluência (intelligibility). O avaliador, nesse caso, é orientado a verificar em que medida o texto-alvo é fluente, ou seja, se as normas linguísticas e socioculturais foram observadas. A resposta dele será expressa na escala mencionada, e essa avaliação pode ser feita até mesmo sem o texto-fonte, pois o enfoque é precisamente o texto-alvo.

Este estudo analisou os resultados da tradução automática, pensando na figura e nas necessidades do tradutor, para verificar especificamente se os sistemas traduzem, com qualidade, combinações especializadas no domínio jurídico; por esse motivo, utilizou os conceitos de "adequação" e "fluência" definidos anteriormente. Nosso intuito foi empreender uma análise qualitativa e descritiva de cada resultado produzido pela tradução automática, considerando que, quanto mais acertos forem verificados, maior utilidade potencial o sistema terá para o tradutor. Há que se mencionar que este trabalho é adequado ao momento presente tendo em vista o estágio de desenvolvimento tecnológico dos sistemas de tradução automática. O estado da arte em tradução automática, o modelo com base em redes neurais 
(NMT), tem se revelado bastante competitivo por apresentar consideravelmente menos erros em relação a seus antecessores (TEIXEIRA, 2018) e por conseguir um desempenho melhor no parâmetro adequação do que no parâmetro fluência (KOEHN; KNOWLES, 2017).

O objetivo deste trabalho se assemelha ao da pesquisa conduzida por Sycz-Opon (2014), que apresentou resultados de tradução automática do gênero textual contratos (no par inglês > polonês) para auxiliar os tradutores a tomarem decisões informadas a respeito da inclusão de sistemas de tradução automática em seu ambiente de trabalho. Entendemos, conforme a autora, que as métricas automáticas ou semiautomáticas de avaliação não se adéquam ao nosso objetivo, pois o que a máquina conta como erro (por ser diferente do gold standard) pode ser uma tradução alternativa e correta. Ademais, a contagem automática de erros pode não refletir o esforço cognitivo demandado pelo tradutor humano para corrigir a tradução automática, ou seja, a métrica pode considerar que certa frase exige esforço significativo de pós-edição quando, na verdade, a percepção do tradutor humano é bem diferente (SYCZ-OPON, 2014).

Por esse motivo, entende-se que a avaliação humana é a mais adequada a este trabalho. Com base em Teixeira (2018), que parte de sua experiência como tradutor de patentes para realizar as avaliações de tradução automática com metodologia e escopo diferentes dos nossos, utilizamos nesta pesquisa a experiência de uma das autoras como bacharel em direito e tradutora de textos jurídicos para a avaliação.

\section{Metodologia}

Tendo em vista que o discurso jurídico é amplo e abrange diversos gêneros textuais (e.g., decisões judiciais, livros acadêmicos, contratos, certidões, escrituras) e diversas subáreas do direito (e.g., direito criminal, direito processual, direito de família, direito internacional), cada qual com terminologia e fraseologia próprias, o primeiro passo deste trabalho foi a seleção de textos para o corpus de análise. Optou-se por selecionar o gênero textual contrato e um tipo específico de cláusula: as cláusulas arbitrais (ou cláusulas compromissórias). Essas cláusulas estabelecem, em geral, que a resolução de qualquer desavença que surgir em decorrência do contrato será por arbitragem, ou seja, a questão não será levada ao poder judiciário. Elas foram escolhidas porque tendem a obedecer a certo padrão de redação, não havendo grande variedade de texto. Elas costumam ser breves, sem grande número de orações intercaladas em comparação a outros tipos de cláusula. Esse foi o primeiro motivo para escolhê-las para esta análise. Como nosso objetivo foi verificar a 
qualidade da tradução das combinações lexicais especializadas, entendeu-se que, caso fossem utilizados textos com frases excessivamente longas e estrutura complexa, tal característica poderia representar uma dificuldade para a ferramenta de tradução automática e interferir na tradução das combinações lexicais especializadas.

As cláusulas selecionadas para compor o corpus foram extraídas de sites de diversas câmaras arbitrais, redigidas em países de língua oficial inglesa e outros países. Não se diferenciou se o texto-fonte foi produzido originalmente em inglês (por falante nativo ou não) ou se é fruto de tradução a partir de qualquer língua. Entende-se que a língua é uma grande instituição democratizante e que cada pessoa que aprende uma língua adquire imediatamente direitos sobre ela - direitos de modificá-la, ignorar partes dela, brincar com ela etc. (CRYSTAL, 2004).

Formou-se, então, um corpus com 18 textos, totalizando 1.039 palavras. Seguindo a metodologia proposta por L'Homme e Bertrand (2000), a primeira etapa consistiu na extração das palavras-chave preliminares. Fizemos isso incluindo o corpus em formato txt na ferramenta de análise de corpus AntConc e extraindo uma lista de palavras-chave, que teve como referência a lista de frequência de palavras do British National Corpus (BNC) para inglês escrito. Uma lista de 30 palavras-chave foi gerada para ser utilizada como indicativo dos termos no nosso corpus que atendem ao critério especificidade. Consideramos que eram termos as palavras resultantes dessa lista que atendiam aos critérios de pertencer à área e à subárea analisada e que possuíam sentido relevante para o projeto.

A segunda etapa foi a extração das combinações lexicais em que as palavras-chave preliminares figuraram (L'HOMME; BERTRAND, 2000). Optou-se por delimitar as colocações lexicais especializadas a serem analisadas àquelas formadas pelo padrão verbo+substantivo, sendo o substantivo considerado o termo (ou palavra-chave) e o verbo, o coocorrente. Essa escolha foi feita por se considerarem de grande relevância os verbos nos domínios especializados (PIMENTEL, 2017). Embora muitos recursos terminológicos priorizem os substantivos e negligenciem outras classes gramaticais, o conhecimento especializado vai além dos objetos e inclui também as ações, representando um desafio para os tradutores. Especificamente em relação ao domínio jurídico, embora muitos tradutores conheçam o significado do verbo "absolver" no sentido de "absolver o réu do crime", podem desconhecer o significado do mesmo verbo na frase "absolver o réu da instância" (PIMENTEL, 2017). Podemos citar, ainda, como mais um exemplo da importância dos verbos, a diferença sutil entre ajuizar, entrar com, impetrar e interpor. Os quatro se referem a 
uma manifestação das partes (autor ou réu) no âmbito de um processo judicial. No entanto, diz-se "ajuizar uma ação" (caráter mais técnico) ou "entrar com uma ação" (caráter menos técnico), mas os outros dois verbos não se aplicam à ação. Diz-se, ainda, "impetrar um mandado de segurança" ou um habeas corpus. O uso desse último verbo é pouco comum para se referir a recurso, que normalmente é acompanhado do verbo interpor (interpor um recurso). Nota-se, portanto, que os verbos têm importância fundamental na linguagem especializada e têm um caráter convencional, o que torna o estudo das combinações lexicais especializadas no padrão verbo+substantivo de grande relevância para os Estudos da Tradução.

Dessa forma, foram selecionados apenas os substantivos presentes na lista de palavraschave gerada. Nesta etapa da metodologia, esses substantivos foram analisados no corpus e, quando verificada sua ocorrência no padrão verbo+substantivo, tal combinação foi considerada para a presente análise. Para fins de exemplificação, embora todos os substantivos em negrito na cláusula a seguir tenham figurado na lista de palavras-chave, apenas aqueles em negrito e sublinhados aparecem no padrão verbo+substantivo, estando o verbo a que se referem apenas sublinhado:

Any dispute, controversy or $\underline{\text { claim }}$ arising out or relating to this contract, or the breach, termination or validity thereof, shall be finally settled by arbitration in accordance with the Arbitration Rules of the Finland Chamber of Commerce.

No exemplo acima, apenas os três substantivos dispute, controversy e claim, juntamente com o verbo a que se referem - to settle - foram extraídos para análise (ou seja, to settle a dispute, to settle a controversy, to settle a claim). Ao final da segunda etapa, obtivemos o conjunto de combinações lexicais especializadas presentes no corpus que continham como termo os substantivos que haviam sido validados como tal e seus coocorrentes verbais.

A terceira etapa pretendeu ampliar o conjunto de combinações lexicais especializadas resultantes da segunda etapa tomando como ponto de partida os coocorrentes nelas verificados para a extração de novas combinações lexicais especializadas. Assim, retornamos ao corpus para localizar os coocorrentes (verbos) do conjunto de combinações lexicais especializadas obtido. Quando tais coocorrentes foram encontrados no padrão verbo+substantivo em combinações que não haviam sido extraídas na segunda etapa, essas novas combinações lexicais especializadas foram incluídas no conjunto para serem analisadas. 
O substantivo que apareceu conectado ao coocorrente foi, então, extraído como novo termo, tendo sido verificada a relação semântica entre esse novo termo e o termo da combinação lexical especializada usada para a nova extração. A título de exemplo, na segunda etapa da metodologia obtivemos, dentre outras, a seguinte combinação (estando o coocorrente apenas

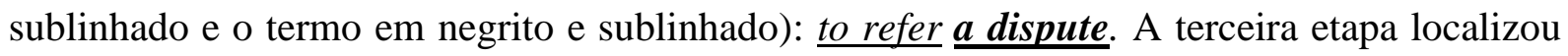
as ocorrências de to refer no corpus que não haviam sido selecionadas e encontrou to refer $\underline{\boldsymbol{a}}$


combinações lexicais especializadas a serem analisadas e, ao final desta etapa, tal conjunto estava completo.

Em seguida, a quarta etapa correspondeu à efetiva tradução automática do corpus constituído inserindo-se cada cláusula selecionada no site do Google Translate ${ }^{5}$ e DeepL ${ }^{6}$. As traduções foram, então, copiadas para um quadro abaixo de seu respectivo texto-fonte com o objetivo de facilitar a análise dos resultados.

Por fim, a quinta etapa consistiu na análise da qualidade dos resultados obtidos para as combinações lexicais especializadas selecionadas. Conforme exposto, entendemos que a definição do objetivo de cada avaliação de qualidade e a própria delimitação do conceito de qualidade - conceitos esses interconectados e, de certa forma, codependentes - são essenciais para a utilidade da avaliação. Posicionamo-nos em relação a tais conceitos partindo da perspectiva do tradutor, que pode vir a usar os sistemas de tradução automática para auxiliá-lo em seu trabalho. Nosso objetivo é verificar em que medida tais sistemas podem ser úteis a ele produzindo traduções que precisem de poucas alterações para serem consideradas finalizadas. Além disso, sendo nosso objeto específico a análise das combinações lexicais especializadas (verbo+substantivo), entendemos que haverá qualidade se houver uma combinação dos parâmetros adequação e fluência. A adequação se justifica como parâmetro escolhido por se referir à própria transferência do significado do texto-fonte para o texto-alvo (CASTILHO et al., 2018) - essencial no caso das cláusulas arbitrais e, de forma geral, em qualquer tradução jurídica. Já a fluência, ao se referir à conformidade com as normas linguísticas e socioculturais, tem razão de ser neste trabalho justamente por termos escolhido tratar de estruturas marcadas por alto grau de convencionalidade, quais sejam, as combinações lexicais especializadas.

A seguir, expomos os resultados obtidos em cada etapa descrita nesta seção, desde a seleção das combinações lexicais especializadas até a análise da qualidade das traduções geradas automaticamente, nosso objetivo final. 


\section{Resultados}

\subsection{Etapa 1: seleção de palavras-chave preliminares}

Conforme descrito anteriormente, as palavras-chave do corpus em análise foram obtidas a partir da lista de palavras-chave gerada por meio da ferramenta AntConc. A Figura 1 revela os resultados obtidos.

Figura 1 - Lista de palavras-chave

$\begin{array}{llllll}\text { \#Keyword Types: } & 82 & & & \\ \text { \#Keyword Tokens: } & 687 & & & \\ \text { \#Search } & \text { Hits: } & 0 & & & \\ 1 & 39 & + & 579.23 & 0.0436 & \text { arbitration } \\ 2 & 15 & + & 257.21 & 0.0258 & \text { xa } \\ 3 & 27 & + & 236.25 & 0.0048 & \text { rules } \\ 4 & 18 & + & 200.35 & 0.0115 & \text { arising } \\ 5 & 23 & + & 171.68 & 0.0026 & \text { shall } \\ 6 & 16 & + & 161.54 & 0.0077 & \text { dispute } \\ 7 & 49 & + & 155.11 & 0.0003 & \text { or } \\ 8 & 11 & + & 153.46 & 0.0159 & \text { mediation } \\ 9 & 14 & + & 150.19 & 0.0092 & \text { accordance } \\ 10 & 9 & + & 113.09 & 0.0114 & \text { termination } \\ 11 & 15 & + & 111.09 & 0.0025 & \text { contract } \\ 12 & 9 & + & 93.35 & 0.007 & \text { disputes } \\ 13 & 6 & + & 85.02 & 0.0098 & \text { arbitrators } \\ 14 & 8 & + & 83.35 & 0.0067 & \text { validity } \\ 15 & 8 & + & 77.71 & 0.0055 & \text { controversy } \\ 16 & 9 & + & 76.68 & 0.0037 & \text { settled } \\ 17 & 6 & + & 71.79 & 0.0079 & \text { thereof } \\ 18 & 8 & + & 71.44 & 0.0042 & \text { chamber } \\ 19 & 8 & + & 67.72 & 0.0035 & \text { relating } \\ 20 & 4 & + & 66.26 & 0.0073 & \text { arbitral } \\ 21 & 5 & + & 62.01 & 0.0073 & \text { arbitrator } \\ 22 & 7 & + & 59.01 & 0.0034 & \text { breach } \\ 23 & 9 & + & 58.52 & 0.0016 & \text { claim } \\ 24 & 4 & + & 58.31 & 0.0069 & \text { hereinafter } \\ 25 & 6 & + & 57.01 & 0.0046 & \text { commerce } \\ 26 & 17 & + & 56.29 & 0.0003 & \text { any } \\ 27 & 6 & + & 53.55 & 0.0038 & \text { submitted } \\ 28 & 7 & + & 52.96 & 0.0024 & \text { connection } \\ 29 & 2 & + & 45.18 & 0.0037 & \text { aise } \\ 30 & 2 & + & 45.18 & 0.0037 & \text { cbma } \\ & 9 & & & & \end{array}$

Fonte: elaboração própria.

A lista foi, então, reduzida para incluir apenas substantivos que atenderam aos critérios de pertencer à área e à subárea analisada e possuir sentido relevante para o projeto. Para ocorrências no plural e no singular, foram considerados apenas o singular. Chegamos aos seguintes termos: arbitration, rule, dispute, mediation, termination, contract, arbitrator, validity, controversy, chamber, breach, claim e commerce. 


\subsection{Etapa 2: extração de combinações lexicais especializadas em que os termos são}

usados

Tendo selecionado os termos, retornamos ao corpus para verificar suas ocorrências no padrão verbo+substantivo e extrair as combinações lexicais especializadas. Obtivemos o seguinte resultado:

Quadro 1 - Termos e coocorrentes

\begin{tabular}{|c|c|}
\hline Termo & Coocorrente \\
\hline arbitration & to administer by \\
\hline arbitrator & to appoint \\
\hline claim & to decide by \\
\hline claim & to determine by \\
\hline claim & to resolve by \\
\hline claim & to settle by \\
\hline claim & to submit to \\
\hline controversy & to decide by \\
\hline controversy & to determine by \\
\hline controversy & to settle by \\
\hline controversy & to submit to \\
\hline controversy & to resolve by \\
\hline dispute & to decide \\
\hline dispute & to determine by \\
\hline dispute & to settle by \\
\hline dispute & to submit to \\
\hline dispute & to refer to \\
\hline dispute & to resolve by \\
\hline rule & to adhere to \\
\hline rule & to deem \\
\hline &
\end{tabular}

Fonte: elaboração própria.

Nem todos os termos obtidos na Etapa 1 foram encontrados no corpus no padrão verbo+substantivo. São eles: mediation, termination, contract, validity, chamber, breach e commerce. Tais termos foram desconsiderados por não servirem para a localização da espécie de combinação lexical especializada selecionada como objeto deste trabalho.

Analisando o Quadro 1, confirmamos o argumento defendido por L'Homme e Bertrand (2000) segundo o qual, diferentemente da língua geral, os coocorrentes na linguagem especializada tendem a se combinar com grupos de unidade terminológica semanticamente relacionadas. Para os dez coocorrentes verbais encontrados, cinco figuraram em combinações lexicais com três termos semanticamente relacionados, como demonstrado no Quadro 2. 
Quadro 2 - Coocorrentes e termos semanticamente relacionados

Fonte: elaboração própria.

\begin{tabular}{|c|c|}
\hline Coocorrente & Termo \\
\hline to decide & claim, controversy, dispute \\
\hline to determine by & claim, controversy, dispute \\
\hline to resolve by & claim, controversy, dispute \\
\hline to settle by & claim, controversy, dispute \\
\hline to submit to & claim, controversy, dispute \\
\hline
\end{tabular}

Os coocorrentes que não estão no Quadro 2 não se combinam com termos semanticamente relacionados, muito provavelmente devido ao tamanho reduzido do nosso corpus, ou seja, não encontramos o padrão verbo+substantivo contendo termos semanticamente relacionados a arbitration, arbitrator e rule.

\subsection{Etapa 3: nova extração de combinações com os coocorrentes selecionados}

A partir dos coocorrentes verificados, retornamos ao corpus para a extração de novas combinações lexicais especializadas, incluindo o coocorrente e os novos termos que não estavam incluídos nas palavras-chave preliminares.

Quadro 3-Coocorrentes e novos termos

\begin{tabular}{|c|c|}
\hline Coocorrente & Novo termo \\
\hline to refer to & question \\
\hline to resolve by & question \\
\hline to submit to & grievance, disagreement \\
\hline to settle & disagreement \\
\hline
\end{tabular}

Fonte: elaboração própria.

Percebe-se, portanto, que mais termos semanticamente relacionados aos expostos no Quadro 2 foram encontrados em combinação lexical especializada no padrão verbo+substantivo. Logo, essa verificação reforça o traço diferencial das combinações lexicais especializadas exposto na Etapa 2. Dessa forma, as combinações lexicais especializadas constantes dos Quadros 1 e 3 formaram o conjunto do objeto de análise.

\subsection{Etapa 4: tradução automática nos sistemas Google Translate e DeepL}

A Etapa 4 consistiu na efetiva tradução de cada cláusula selecionada no site do Google Translate e no site do DeepL. 


\subsection{Etapa 5: análise das traduções}

A Etapa 5 consistiu na análise humana das traduções resultantes, caso a caso, seguindo os parâmetros de adequação e fluência. A adequação foi considerada como a transferência correta do significado do texto-fonte para o texto-alvo, e a fluência foi entendida como a observância às normas linguísticas e socioculturais da língua-alvo. Nos Quadros 4 a 9 estão transcritas algumas cláusulas e suas traduções em formato de quadro comparativo em que a primeira linha corresponde ao texto-fonte (TF); a segunda, à tradução obtida com o Google Translate (TG); e a terceira, à tradução produzida pelo DeepL (TD). Nem todos os textosfonte e suas traduções correspondentes foram transcritos já que a tradução de algumas cláusulas não suscitou nenhuma observação pela avaliadora. Além disso, houve repetição dos mesmos erros nas traduções de cláusulas diferentes, estando, nesse caso, apenas uma delas aqui transcrita. As combinações lexicais especializadas que haviam sido selecionadas foram marcadas em negrito - tanto no texto-fonte como nas traduções.

Quadro 4 - Comparação das traduções de to submit/to settle a dispute/controversy/claim

\begin{tabular}{|c|l|}
\hline TF & $\begin{array}{l}\text { Any dispute, controversy, or claim arising out of or in connection with this contract, or the breach, } \\
\text { termination or validity thereof, shall be submitted to the Chartered Institute of Arbitrators (CIArb) } \\
\text { and settled by final and binding arbitration in accordance with the CIArb Arbitration Rules. } \\
\text { Judgment on any award issued under this provision may be entered by any court of competent } \\
\text { jurisdiction. }\end{array}$ \\
\hline TG & $\begin{array}{l}\text { Qualquer disputa, controvérsia ou reclamação decorrente ou relacionada a este contrato, ou a } \\
\text { violação, rescisão ou validade do mesmo, deverá ser submetida ao Instituto de Arbitragem } \\
\text { Chartered (CIArb) e resolvida por arbitragem final e vinculante, de acordo com o CIArb. Regras } \\
\text { de Arbitragem. O julgamento de qualquer prêmio emitido sob esta provisão pode ser inserido por } \\
\text { qualquer tribunal de jurisdição competente. }\end{array}$ \\
\hline TD & $\begin{array}{l}\text { Qualquer disputa, controvérsia ou reclamação que surja no âmbito ou relacionada com este } \\
\text { contrato, ou a violação, rescisão ou validade do mesmo, deve ser submetida ao Chartered Institute } \\
\text { of Arbitration (CIArb) e resolvida por arbitragem final e vinculativa de acordo com a CIArb. } \\
\text { Regras de Arbitragem. A sentença de qualquer prémio emitido ao abrigo da presente disposição } \\
\text { pode ser proferida por qualquer tribunal de jurisdição competente. }\end{array}$ \\
\hline
\end{tabular}

Fonte: corpus compilado por Borges e Pimentel.

- Adequação: claim foi traduzido em ambas as ferramentas por reclamação, que é um termo com significado muito particular na área do Direito - uma peça processual que pode ser proposta na justiça em casos específicos e, também, muito relacionada ao direito do trabalho. Tanto é assim que o Dicionário de Direito, Economia e Contabilidade, de autoria de Castro (2013), não prevê essa tradução dentre as sugestões de tradução para claim. Para o público em geral, a escolha atende à adequação por transferir o significado genérico da palavra; porém, por referir-se a um domínio especializado, a adequação não foi atendida. 
- Fluência: ambas atendem ao critério fluência.

Quadro 5 - Comparação das traduções de to submit a dispute/claim/grievance e to administer an arbitration

\begin{tabular}{|c|l|}
\hline TF & $\begin{array}{l}\text { Labor - Any dispute, claim, or grievance arising from or relating to the interpretation or } \\
\text { application of this agreement shall be submitted to arbitration administered by the American } \\
\text { Arbitration Association under its Labor Arbitration Rules. The parties further agree to accept the } \\
\text { arbitrator's award as final and binding on them. }\end{array}$ \\
\hline TG & $\begin{array}{l}\text { Trabalho - Qualquer disputa, reclamação ou queixa decorrente ou relacionada à interpretação ou } \\
\text { aplicação deste contrato deverá ser submetida à arbitragem administrada pela Associação } \\
\text { Americana de Arbitragem sob as Regras de Arbitragem Trabalhista. As partes concordam ainda em } \\
\text { aceitar o prêmio do árbitro como final e obrigatório para ele. }\end{array}$ \\
\hline TD & $\begin{array}{l}\text { Trabalhista - Qualquer disputa, reclamação ou reclamação decorrente ou relacionada à } \\
\text { interpretação ou aplicação deste contrato deverá ser submetida à arbitragem administrada pela } \\
\text { American Arbitration Association sob suas Regras de Arbitragem Trabalhista. As partes também } \\
\text { concordam em aceitar a decisão do árbitro como final e vinculativa para elas. }\end{array}$ \\
\hline
\end{tabular}

Fonte: corpus compilado por Borges e Pimentel.

- Adequação: por se tratar de uma cláusula de arbitragem trabalhista, consideramos a tradução de claim (ver Quadro 4) por reclamação adequada. Muito embora tecnicamente seja a peça processual que dá início a uma ação trabalhista, pode ser entendida como controvérsia trabalhista em um sentido amplo nessa cláusula. Por outro lado, traduzir o mesmo termo por queixa, como na linha TG, é inadequado no contexto trabalhista, pois queixa é a peça processual que dá início a uma espécie de ação penal no direito brasileiro.

- Fluência: na linha TD, a tradução de dois termos diferentes no texto-fonte (claim, grievance) por um mesmo termo repetido no texto-alvo (reclamação) não se revela fluente, uma tradução natural. Contudo, a combinação resultante (submeter reclamação) atende ao critério fluência. 
Quadro 6 - Comparação das traduções de to submit a dispute/disagreement e to settle a disagreement

\begin{tabular}{|c|l|}
\hline TF & $\begin{array}{l}\text { Any dispute or disagreement arising out of or in connection with the present contract shall be } \\
\text { submitted to mediation in accordance with the Mediation Rules of the Association Française } \\
\text { d'Arbitrage(1). } \\
\text { In the absence of agreement within six months following the appointment of a Mediator, the parties } \\
\text { agree on settling the disagreement in accordance with the Arbitration Rules of the Association } \\
\text { Française d'Arbitrage. }\end{array}$ \\
\hline TG & $\begin{array}{l}\text { Qualquer litígio ou desacordo decorrente ou relacionado com o presente contrato será submetido à } \\
\text { mediação de acordo com as Regras de Mediação da Association Française d'Arbitrage (1). } \\
\text { Na ausência de acordo no prazo de seis meses após a nomeação de um mediador, as partes } \\
\text { concordam em resolver o desacordo de acordo com as Regras de Arbitragem da Association } \\
\text { Française d'Arbitrage. }\end{array}$ \\
\hline TD & $\begin{array}{l}\text { Qualquer litígio ou desacordo resultante ou relacionado com o presente contrato deve ser } \\
\text { submetido a mediação de acordo com o Regulamento de Mediação da Association Française } \\
\text { d'Arbitrage(1). } \\
\text { Na ausência de acordo no prazo de seis meses após a nomeação de um Mediador, as partes acordam } \\
\text { em resolver o diferindo de acordo com as Regras de Arbitragem da Association Française } \\
\text { d'Arbitrage. }\end{array}$ \\
\hline
\end{tabular}

Fonte: corpus compilado por Borges e Pimentel.

- Adequação: ambas atendem ao critério adequação.

- Fluência: embora o significado da palavra "diferindo" na linha TD transmita o significado adequado, ele não é usual nesse tipo de cláusula, sendo, portanto, pouco fluente.

Quadro 7 - Comparação das traduções de to resolve a dispute/controversy/claim/question

TF $\quad$ Any dispute, controversy or claim arising out of, relating to or in connection with this contract, including any question regarding its existence, validity or termination, shall be resolved by arbitration in accordance with the ACICA Arbitration Rules.

TG Qualquer disputa, controvérsia ou reivindicação decorrente de, relacionada a ou relacionada a este contrato, incluindo qualquer questão relacionada a sua existência, validade ou rescisão, deverá ser resolvida por arbitragem, de acordo com as Regras de Arbitragem da ACICA.

TD Qualquer disputa, controvérsia ou reclamação decorrente, relacionada ou relacionada a este contrato, incluindo qualquer dúvida sobre sua existência, validade ou rescisão, será resolvida por arbitragem de acordo com o Regulamento de Arbitragem da ACICA.

Fonte: corpus compilado por Borges e Pimentel.

- Adequação: sobre claim, ver Quadro 4. A tradução de question por dúvida na linha TD não atende ao critério adequação, pois o que há não são dúvidas, mas questões no sentido de problemas, impasses, controvérsias.

- Fluência: ambas atendem ao critério fluência. 
Quadro 8 - Comparação das traduções de to settle a dispute, to appoint an arbitrator, to deem a rule

\begin{tabular}{|c|l|}
\hline TF & $\begin{array}{l}\text { All disputes - included those of not contractual nature - arising out of, related or connected to this } \\
\text { agreement shall be settled by arbitration under the Rules of the Milan Chamber of Arbitration (the } \\
\text { Rules), by a sole arbitrator / three arbitrators **, appointed in accordance with the Rules, which are } \\
\text { deemed to be incorporated by reference into this clause. }\end{array}$ \\
\hline TG & $\begin{array}{l}\text { Todas as disputas - incluindo aquelas de natureza não contratual - decorrentes, relacionadas ou } \\
\text { conectadas a este acordo serão resolvidas por arbitragem sob as Regras da Câmara de Arbitragem } \\
\text { de Milão (as Regras), por um único árbitro / três árbitros **, nomeados de acordo com as Regras, } \\
\text { que são consideradas incorporadas por referência nesta cláusula. }\end{array}$ \\
\hline TD & $\begin{array}{l}\text { Todos os litígios - inclusive aqueles de natureza não contratual - oriundos, relacionados ou } \\
\text { vinculados ao presente contrato serão resolvidos por arbitragem de acordo com o Regulamento da } \\
\text { Câmara de Arbitragem de Milão (o Regulamento), por um único árbitro / três árbitros **, } \\
\text { nomeados de acordo com o Regulamento, que se presumem incorporados por referência a esta } \\
\text { cláusula. }\end{array}$ \\
\hline
\end{tabular}

Fonte: corpus compilado por Borges e Pimentel.

- Adequação: ambas atendem ao critério adequação.

- Fluência: embora a escolha do verbo presumir na linha TD atenda à fluência, tal verbo se refere ao Regulamento, devendo obedecer à norma linguística de concordância em número.

Quadro 9 - Comparação das traduções de to refer to/determine/decide a dispute/controversy/claim

TF $\quad$ Any dispute, controversy or claim arising under, out of or relating to this contract and any subsequent amendments of this contract, including, without limitation, its formation, validity, binding effect, interpretation, performance, breach or termination, as well as non-contractual claims, shall be referred to and finally determined by arbitration in accordance with the WIPO Arbitration Rules. The arbitral tribunal shall consist of [a sole arbitrator][three arbitrators]. The place of arbitration shall be [specify place]. The language to be used in the arbitral proceedings shall be [specify language]. The dispute, controversy or claim shall be decided in accordance with the law of [specify jurisdiction].

TG Qualquer controvérsia, controvérsia ou reclamação decorrente, fora ou relacionada a este contrato e quaisquer emendas subsequentes a este contrato, incluindo, sem limitação, sua formação, validade, efeito vinculante, interpretação, desempenho, violação ou rescisão, bem como reclamações contratuais, serão referidas e finalmente determinadas por arbitragem, de acordo com as Regras de Arbitragem da OMPI. O tribunal arbitral consistirá de [um único árbitro] [três árbitros]. O local da arbitragem será [especificar local]. O idioma a ser usado no procedimento arbitral deve ser [especificar idioma]. A disputa, controvérsia ou reclamação será decidida de acordo com a lei de [especificar jurisdição].

TD Qualquer disputa, controvérsia ou reclamação decorrente, oriunda ou relacionada a este contrato e a quaisquer alterações posteriores do mesmo, incluindo, sem limitação, sua formação, validade, efeito vinculante, interpretação, execução, violação ou rescisão, bem como reclamações extracontratuais, serão submetidas e decididas em caráter final por arbitragem de acordo com o Regulamento de Arbitragem da OMPI. O tribunal arbitral será composto por [um único árbitro] [três árbitros]. O local da arbitragem será [especificar local]. O idioma a ser utilizado no procedimento arbitral será [especificar idioma]. A disputa, controvérsia ou demanda será decidida de acordo com a lei de [especificar jurisdição].

Fonte: corpus compilado por Borges e Pimentel. 
- Adequação: Sobre claim, ver Quadro 4.

- Fluência: Na linha TG, a tradução de dois termos diferentes no texto-fonte (dispute, controversy) por um mesmo termo repetido no texto-alvo (controvérsia) não se revela fluente, pois não é natural em uma enumeração a repetição de dois termos idênticos em sequência. Tal repetição causa grande estranhamento ao leitor, parecendo ser, inclusive, um erro do texto. Além disso, a tradução de to refer, combinado com dispute, controversy e claim, para referir não se revela fluente, sendo mais usual o verbo submeter.

\section{Conclusão}

Neste trabalho pretendemos avaliar as traduções automáticas fornecidas pelos sistemas Google Translate e DeepL para as combinações lexicais especializadas no padrão verbo+substantivo no domínio jurídico, especificamente em cláusulas arbitrais. As combinações foram selecionadas com base na metodologia utilizada por L'Homme e Bertrand (2000). Iniciou-se com a extração de palavras-chave preliminares no corpus a serem validadas

38 como termos. A partir dos termos, seguimos para a primeira extração de combinações lexicais especializadas quando tais termos apareceram no padrão verbo+substantivo no corpus, sendo o verbo o coocorrente e o substantivo o termo. Em seguida, localizamos os coocorrentes no corpus e obtivemos novas combinações lexicais especializadas contendo termos que não figuraram na lista de palavras-chave inicial. O corpus foi, então, traduzido de forma automática, e o resultado foi avaliado de forma qualitativa quanto aos critérios adequação e fluência.

As avaliações das combinações lexicais revelaram traduções bastante satisfatórias feitas pelos sistemas de tradução automática selecionados para o presente trabalho. $\mathrm{O}$ resultado se mostra mais confiável do que poderia se esperar há poucos anos, especialmente para um texto de caráter técnico em que a terminologia é fundamental. Foram notadas mais questões relativas à adequação do que à fluência: as traduções atenderam ao caráter de naturalidade do texto-alvo apresentando combinações lexicais especializadas bastante fluentes, mas por vezes revelaram falta de precisão terminológica, que é essencial em uma tradução de texto técnico. Em alguns casos, apesar de o termo se mostrar inadequado (e.g., queixa), a combinação termo+coocorrente (e.g., submeter queixa) se revelou fluente.

Embora não tenha sido o objetivo específico deste trabalho uma comparação entre os sistemas, mais observações críticas foram feitas sobre as traduções resultantes do DeepL do 
que a respeito daquelas provenientes do Google Translate. Além disso, no que se refere ao desempenho de ambas nos parâmetros adequação e fluência, ressaltamos que o resultado obtido está alinhado com pesquisas na área de tradução automática baseada em redes neurais (NMT) que indicam que esse tipo de tecnologia tem um desempenho melhor no parâmetro adequação do que no parâmetro fluência (KOEHN; KNOWLES, 2017). Ainda em relação à precisão terminológica, houve ocorrências de tradução de termos diferentes na língua-fonte para o mesmo termo na língua-alvo em uma mesma frase, o que muitas vezes é inadequado para textos técnicos. Esse resultado contraria a percepção bastante comum de que a tradução automática produz resultados consistentes, sendo mais adequada à linguagem técnica. A consistência terminológica é, na verdade, um problema conhecido dos sistemas de tradução automática e, em especial, daqueles baseados em redes neurais (NMT), quando comparados ao paradigma tecnológico anterior de base estatística. Para superá-lo, há iniciativas de combinações de ambos os modelos tecnológicos, além da possibilidade de associar os sistemas a glossários e memórias de tradução.

Reconhecemos que a avaliação feita por apenas um indivíduo pode estar sujeita a preferências meramente pessoais e sub-representar a convencionalidade de um domínio técnico da língua, como a linguagem jurídica analisada. Insistimos, contudo, que a avaliação humana é a mais adequada para revelar o potencial dos sistemas de tradução automática para o uso pelos tradutores em seus ambientes de trabalho. Não foram incluídos mais avaliadores meramente por limitações temporais e financeiras, pois defendemos veementemente que o saber dos avaliadores e seu tempo devem ser devidamente remunerados. Buscamos, portanto, empreender uma amostra do que poderia ser uma avaliação mais abrangente.

Reconhecemos, ainda, que a metodologia empregada apresenta limitações para ser aplicada em larga escala, pois demanda tempo considerável. Embora tenhamos considerado os resultados das traduções automáticas bastante satisfatórios, seria necessário um volume maior de textos, uma variedade maior de gêneros textuais jurídicos e, logo, mais combinações lexicais especializadas para uma melhor avaliação da qualidade das traduções fornecidas pelos sistemas analisados.

Tendo avaliado as combinações lexicais especializadas compostas por verbo+substantivo extraídas de cláusulas arbitrais, espera-se ter conseguido com este trabalho apresentar subsídios para o tradutor decidir a respeito do potencial dos sistemas de tradução automática como mais uma ferramenta a seu dispor. O mercado de tradução está cada vez 
mais dinâmico e do tradutor se exige cada vez mais produtividade. Por isso, consideramos que a tradução automática pode ser uma ferramenta útil no dia a dia do profissional.

\section{REFERÊNCIAS}

ARNOLD, Doug. Why translation is difficult for computers. In: SOMERS, Harold (Ed.). Computers and translation: a translator's guide. Amsterdam: John Benjamins, 2003. p. 119142. doi: https://doi.org/10.1075/btl.35.11arn

CASTILHO, Sheila; O'BRIEN, Sharon. Acceptability of machine-translated content: A multi-language evaluation by translators and end-users. Linguistica Antverpiensia - New Series: Themes in Translation Studies, v. 16, p. 120-136, 2017. Disponível em: https://lanstts.uantwerpen.be/index.php/LANS-TTS/article/view/430. Acesso em: 26 dez. 2019.

CASTILHO, Sheila; DOHERTY, Stephen; GASPARI, Federico; MOORKENS, Joss. Approaches to Human and Machine Translation Quality Assessment. In: MOORKENS, Joss; CASTILHO, Sheila; GASPARI, Federico; DOHERTY, Stephen (Ed.). Translation quality assessment: From principles to practice. Cham: Springer, 2018. p. 9-38. doi: https://doi.org/10.1007/978-3-319-91241-7_2

CASTRO, Marcílio M. Dicionário de Direito, Economia e Contabilidade - PortuguêsInglês/Inglês-Português. Rio de Janeiro: Forense, 2013.

CONSTANT, Mathieu et al. Multiword expression processing: A survey. Computational Linguistics, v. 43, n. 4, p. 1-92, 2017. Disponível em: https://www.mitpressjournals.org/doi/full/10.1162/COLI_a_00302. Acesso em: 29 dez. 2019. doi: https://doi.org/10.1162/COLI_a_00302

CRYSTAL, David. The language revolution. Cambridge/Malden: Polity Press, 2004.

PÉREZ-HERNÁNDEZ, Chantal; ORTIZ, Antonio Moreno; FABER, Pamela. Lexicografia Computacional y Lexicografia de Corpus. Revista Española de Lingüística Aplicada: Panorama de la investigación em lingúistica informática, v. 1, p. 175-214, 1999. Disponível em:

https://www.researchgate.net/publication/28106208_Lexicografia_computacional_y_lexicogr afia_de_corpus. Acesso em: 26 dez. 2019.

FIEDERER, Rebecca; O'BRIEN, Sharon. Quality and machine translation: A realistic objective? The Journal of Specialized Translation, v. 11, p. 52-74, 2009. Disponível em: https://www.jostrans.org/issue11/art_fiederer_obrien.pdf. Acesso em: 26 dez 2019.

FROTA, Maria Paula. Erros e lapsos de tradução: um tema para o ensino. Cadernos de Tradução, Florianópolis, v. 1, n. 17, p. 141-156, 2006. Disponível em: https://periodicos.ufsc.br/index.php/traducao/article/view/6859. Acesso em: $26 \operatorname{dez} 2019$.

HASSAN, Hany et al. Achieving human parity on automatic Chinese to English news translation. Relatório da Microsoft. 2018. Disponível em: https://www.microsoft.com/en- 
us/research/publication/achieving-human-parity-on-automatic-chinese-to-english-newstranslation. Acesso em: $10 \mathrm{dez} 2019$.

HEID, Ulrich. On the way words work together - Topics in lexical combinatorics. In: MARTIN, Willy et al. (Org.). Proceedings of the Euralex '94. Amsterdam: EURALEX, 2014. p. 226-257.

KOEHN, Philipp; KNOWLES, Rebecca. Six challenges for neural machine translation. In: Proceedings of the First Workshop on Neural Machine Translation. Vancouver (Canadá), 2017. p. 28-39. doi: https://doi.org/10.18653/v1/W17-3204

L'HOMME, Marie-Claude; BERTRAND, Claudine. Specialized lexical combinations: Should they be described as collocations or in terms of selectional restrictions. In: EVERT, Stefan et al. Proceedings of the Ninth Euralex International Congress. Stuttgart: EURALEX, 2000. p. 497-506.

MARTÍN, Beatriz. Translations quality assessment of google translate and microsoft bing translation. 2016. 45f. TCC (Graduação em Estudos Ingleses) - Faculdade de Filosofia e Letras, Departamento de Filologia Inglesa, Universidad de Valladolid, Valladolid, 2017. Disponível https://uvadoc.uva.es/bitstream/handle/10324/22596/TFG_F_2017_7.pdf;jsessionid=153C847 43864859CEF93398BDB9D818A? sequence=1. Acesso em: $26 \mathrm{dez} 2019$.

MUNDAY, Jeremy. Introducing translation studies: Theories and applications. 3. ed. Londres: Routledge. 2012. doi: https://doi.org/10.4324/9780203121252

MUZII, Luigi. Quality assessment and economic sustainability of translation. Rivista internazionale di técnica della traduzione, v. 9, p. 15-38, 2006. Disponível em: https://www.openstarts.units.it/bitstream/10077/2891/1/ritt9_05muzii.pdf. Acesso em: 27 dez. 2019.

ORENHA, Adriane. Unidades fraseológicas especializadas: colocações e colocações estendidas em contratos sociais e estatutos sociais traduzidos no modo juramentado e nãojuramentado. 2009. 290f. Tese (Doutorado em Estudos Linguísticos) - Instituto de Biociências, Letras e Ciências Exatas, Universidade Estadual Paulista, São José do Rio Preto, $2009 . \quad$ Disponível em: https://repositorio.unesp.br/bitstream/handle/11449/103524/orenha_a_dr_sjrp.pdf?sequence= 1\&isAllowed=y. Acesso em: 27 dez. 2019.

PIMENTEL, Janine. Specialized verbs and specialized uses of verbs in a comparable corpus of judgments produced in Canada, Portugal and Brazil. In: DROUIN, Patrick et al. (Org.). Multiple perspectives on terminological variation. Amsterdam/Filadélfia: John Benjamins, 2017. p. 109-130. doi: https://doi.org/10.1075/tlrp.18.05pim

RAMISCH, Carlos. Multi-word terminology extraction for domain-specific documents. Advisor: Christian Boitet. 2009. 77f. Mémoire (Master de Recherche en Infromatique Option Intelligence Artificielle et Web) - Institut National Polytechnique de Grenoble, Grenoble, 2009. Disponível em: 
https://pdfs.semanticscholar.org/79b6/a43105df36fe6e507adc8fa67dfa4d9f9d19.pdf?_ga=2.5 4638955.1171411335.1577447828-749771379.1577447828. Acesso em: 27 dez. 2019.

SAG, Ivan A.; BALDWIN, Timothy; BOND, Francis; COPESTAKE, Ann; FLICKINGER, Dan. Multiword expressions: A pain in the neck for NLP. In: International Conference on Intelligent Text Processing and Computational Linguistics, 3., 2002, Cidade do México. Proceedings... Cidade do México: [s.e.], 2002. p. 1-15. doi: https://doi.org/10.1007/3540-45715-1_1

SYCZ-OPOŃ, Joanna. Machine translation - Can it assist in professional translation of contracts?, International Journal of Legal Discourse, v. 20, p. 81-200, 2014. Disponível em: https://pressto.amu.edu.pl/index.php/cl/article/view/6524. Acesso em: 27 dez. 2019.

TAGNIN, Stella. Os corpora: Instrumentos de auto-ajuda para o tradutor. Cadernos de Tradução, Florianópolis, v.9, n. 9, p. 191-213, 2002. Disponível em: https://periodicos.ufsc.br/index.php/traducao/article/view/5986/5690. Acesso em: $27 \mathrm{dez}$. 2019.

TEIXEIRA, Michel. O jogo da avaliação: um estudo prático sobre tradução automática. 2018. 134f. Dissertação (Mestrado em Estudos da Linguagem) - Programa de PósGraduação em Estudos da Linguagem, Pontifícia Universidade Católica do Rio de Janeiro, Rio de Janeiro, 2018. Disponível em: https://www.maxwell.vrac.puc42 rio.br/41711/41711.PDF. Acesso em: 27 dez. 2019.

VILLAVICENCIO, Aline; RAMISCH, Carlos. Chutando o balde ou batendo as botas? Processamento de linguagem natural e expressões multipalavra na linguagem cotidiana e científica. In: PERNA, Cristina; DELGADO, Heloisa; FINATTO, Maria José (Org.) Linguagens especializadas em corpora modos de dizer e interfaces de pesquisa. Porto Alegre: EDIPUCRS, 2010. p. 29-49.

WAQUIL, Marina. Tradução de textos especializados: unidades fraseológicas especializadas e técnicas tradutórias. 2013. 206f. Dissertação (Mestrado em Letras) - Programa de PósGraduação em Letras, Universidade Federal do Rio Grande do Sul, Porto Alegre, 2013. Disponível

em: https://www.lume.ufrgs.br/bitstream/handle/10183/72737/000883531.pdf?sequence=1\&isAll owed=y. Acesso em: 27 dez. 2019.

WU, Yonghui et al. Google's neural machine translation system: Bridging the gap between human and machine translation. ArXiv, p. 1-23, 2016. Disponível em: https://arxiv.org/pdf/1609.08144.pdf. Acesso em: 10 dez. 2019.

\footnotetext{
${ }^{1}$ Original: "human parity" (HASSAN et al., 2018, p. 2).

2 "Bridging the gap between human and machine translation" (WU et al., 2016, p. 1).

${ }^{3}$ A expressão pain in the neck é um trocadilho. Ela significa que algo é difícil e dá muito trabalho, característica que os autores atribuem ao tratamento das expressões multipalavras tais como pain in the neck.

4 "An integration of the features and characteristics that determine the extent to which output satisfies the customer's needs" (MUZZI, 2006, p. 19).

${ }^{5}$ Google Translate: https://translate.google.com.br/?hl=pt-BR.

${ }^{6}$ DeepL: https://www.deepl.com/translator.
} 


\section{NOTA DOS AUTORES}

Thais Miranda e BORGES - Especialista em Técnicas, Práticas e Estudos da Tradução Inglês-Português (2019) e Bacharel em Direto (2013) pela Pontifícia Universidade Católica do Rio de Janeiro. Pesquisadora autônoma. Rio de Janeiro, Rio de Janeiro, Brasil.

ORCID: https://orcid.org/0000-0001-7092-7424

Currículo acadêmico: http://lattes.cnpq.br/0270905497716168

E-mail: mirandaeborges.thais@gmail.com

Janine Maria Mendonça PIMENTEL - Professora adjunta I na Universidade Federal do Rio de Janeiro. Doutora em Estudos de Tradução (2012) pela Université de Montréal, Canada. Mestre em Lexicologia, Lexicografia e Terminologia (2007) e graduada em Estudos Ingleses e Alemães (2003) pela Universidade Nova de Lisboa, Portugal. Realizou estágio pós-doutoral (2013-2014) na Pontifícia Universidade Católica do Rio de Janeiro. Universidade Federal do Rio de Janeiro, Faculdade de Letras, Departamento de Anglo-Germânicas. Rio de Janeiro, Rio de Janeiro, Brasil.

ORCID: https://orcid.org/0000-0001-6576-9898

Currículo acadêmico: http://lattes.cnpq.br/7411620486443897

E-mail: janine.m.pimentel@gmail.com 